\title{
Correction to: Long-term outcomes of patients with early gastric cancer found to have lesions for which endoscopic treatment is not indicated on histopathological evaluation after endoscopic submucosal dissection
}

\author{
Takafumi Yano $^{1} \cdot$ Kenji Ishido $^{1} \cdot$ Satoshi Tanabe $^{2} \cdot$ Takuya Wada $^{1} \cdot$ \\ Mizutomo Azuma $^{1}$. Natsuko Kawanishi ${ }^{1} \cdot$ Sakiko Yamane $^{1} \cdot$ Akinori Watanabe $^{1}$. \\ Chikatoshi Katada $^{1} \cdot$ Wasaburo Koizumi ${ }^{1}$
}

Published online: 7 December 2017

(C) Springer Science+Business Media, LLC 2017

\section{Correction to: Surg Endosc DOI 10.1007/}

s00464-017-5809-1

In Table 1, the second item in the right-hand column "Extraindication" should be changed to "Out of indication". The correct version of Table 1 is displayed.

In Figure 1, the illustration on the left should be exchanged with that on the right. Also, in the caption's third line, " $p=0.010$ " should be changed to " $p<0.001$ ". The correct version of Fig. 1 is displayed.

In Figure 2, the illustration on the left should be exchanged with that on the right. The correct version of Fig. 2 is displayed.

The online version of the original article can be found under doi:10.1007/s00464-017-5809-1

Takafumi Yano

yano1371@kitasato-u.ac.jp

1 Department of Gastroenterology, Kitasato University School of Medicine, 1-15-1 Kitasato, Minami-ku, Sagamihara, Kanagawa 252-0374, Japan

2 Research and Development Center for New Frontier, Kitasato University School of Medicine, Sagamihara, Japan 
Table 1 Classification of indications for endoscopic submucosal dissection as defined by the Japan Gastroenterological Endoscopy Society (JGES) and the Japanese Gastric Cancer Association (JGCA)

\begin{tabular}{lllll}
\hline Depth of invasion & Ulceration & Differentiated-type & & Undifferentiated-type \\
\hline Clinically diagnosed T1a & $\mathrm{UL}(-)$ & $\leq 2 \mathrm{~cm}$ & $>2 \mathrm{~cm}$ & $\leq 2 \mathrm{~cm}$ \\
& & Absolute indication & Expanded indication & Expanded indication \\
& $\mathrm{UL}(+)$ & $\leq 3 \mathrm{~cm}$ & $>3 \mathrm{~cm}$ & Any size \\
& & Expanded indication & Out of indication & Out of indication \\
Clinically diagnosed T1b & $\mathrm{UL}(-) /(+)$ & Any size & & \\
& & Out of indication & & \\
\hline
\end{tabular}

\section{(A) Overall survival}

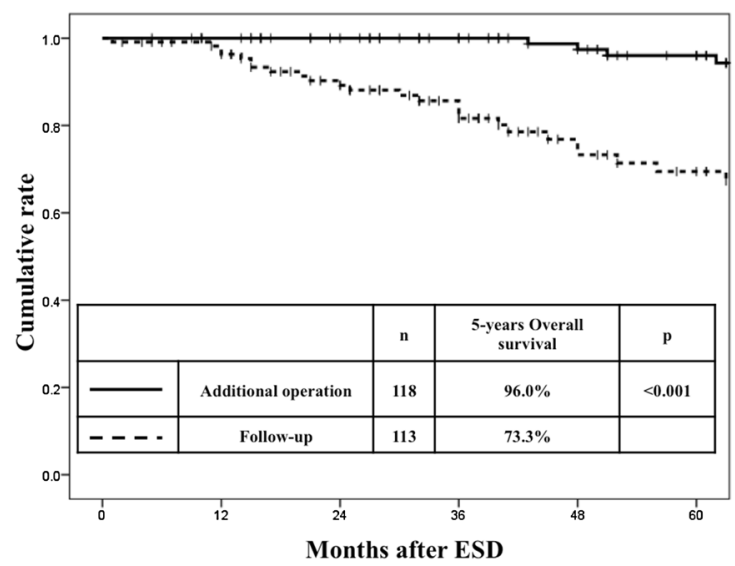

Fig. 1 A Overall survival. The 5-year overall survival rate was significantly higher in the additional operation group $(96.0 \%)$ than in the follow-up group $(73.3 \%, p<0.001)$. B Cause-specific survival.

(A) Overall survival (75 years or older)

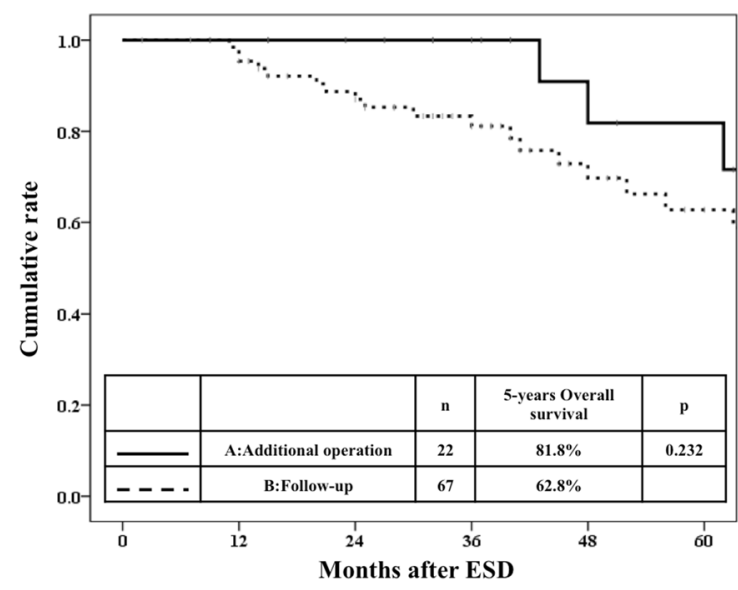

Fig. 2 A Overall survival. Among elderly patients 75 years or older, the 5-year overall survival rate was slightly lower in the followup group $(62.8 \%)$ than in the additional operation group $(81.8 \%$, $p=0.232$ ). B Cause-specific survival. Among elderly patients
(B) Cause-specific survival

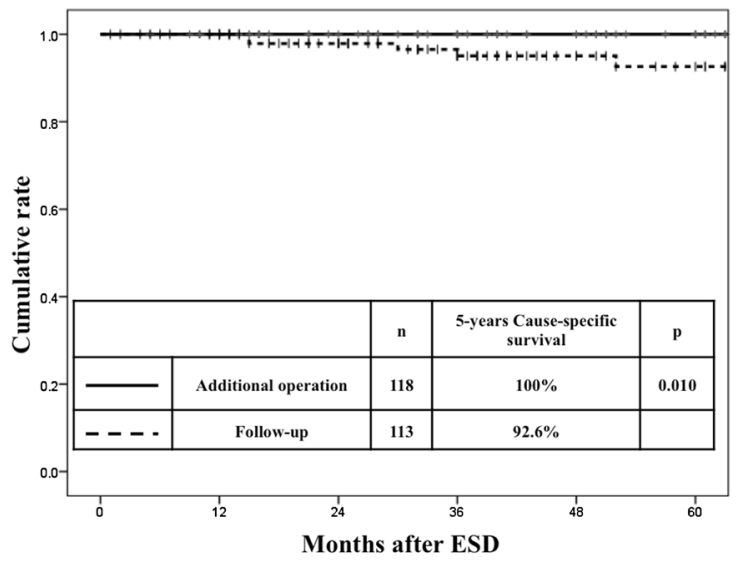

The 5-year cause-specific survival rate was significantly higher in the additional operation group (100\%) than in the follow-up group $(92.6 \%, p<0.001)$

\section{(B) Cause-specific survival (75 years or older)}

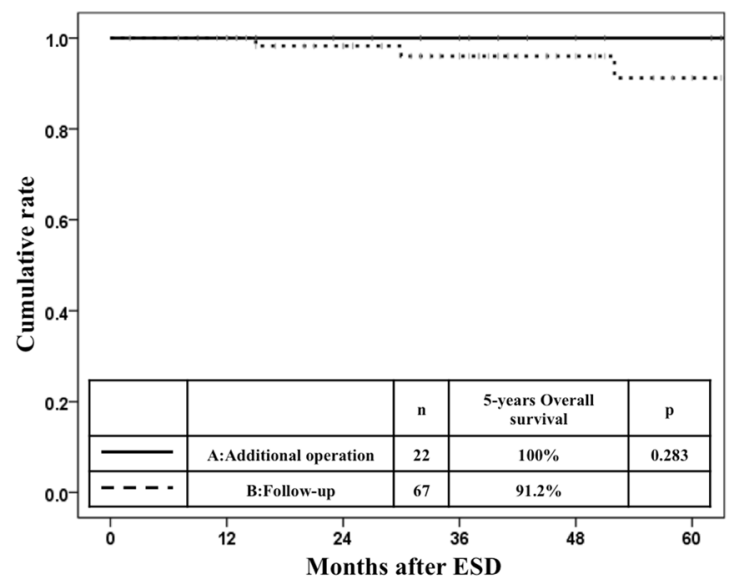

75 years or older, the 5-year cause-specific survival rate did not differ significantly between the additional operation group (100\%) and the follow-up group $(91.2 \%, p=0.283)$ 